\title{
Discovery of $\delta$ Scuti Pulsations in the Young Hybrid Debris Disk Star HD 156623
}

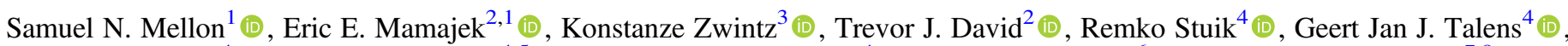 \\ Patrick Dorval $^{4}$ (D), Olivier Burggraaff ${ }^{4,5}$ (D), Matthew A. Kenworthy ${ }^{4}$ (D), John I. Bailey, III ${ }^{6}$ (D), Blaine B. D. Lomberg ${ }^{7,8}$ (D), \\ Rudi B. Kuhn ${ }^{7}$ (D), Michael J. Ireland ${ }^{9}$ (D), and Steven M. Crawford ${ }^{7,10}$ (D) \\ ${ }^{1}$ Department of Physics \& Astronomy, University of Rochester, 500 Wilson Boulevard, Rochester, NY 14627, USA; smellon@ur.rochester.edu \\ ${ }^{2}$ Jet Propulsion Laboratory, California Institute of Technology, M/S 321-100, 4800 Oak Grove Drive, Pasadena, CA 91109, USA \\ 3 Institut für Astro- und Teilchenphysik, Universität Innsbruck, Technikerstrasse 25/8, A-6020 Innsbruck, Austria \\ ${ }^{4}$ Leiden Observatory, Leiden University, P.O. Box 9513, 2300 RA Leiden, The Netherlands \\ ${ }^{5}$ Institute of Environmental Sciences (CML), Leiden University, P.O. Box 9518, 2300 RA Leiden, The Netherlands \\ ${ }^{6}$ Department of Physics, University of California at Santa Barbara, Santa Barbara, CA 93106, USA \\ ${ }^{7}$ South African Astronomical Observatory, Observatory Road, Observatory Cape Town, 7700 Cape Town, South Africa \\ ${ }^{8}$ Department of Astronomy, University of Cape Town, Rondebosch, 7700 Cape Town, South Africa \\ ${ }^{9}$ Research School of Astronomy and Astrophysics, Australian National University, Canberra, ACT 2611, Australia \\ ${ }^{10}$ Space Telescope Science Institute, 3700 San Martin Drive, Baltimore, MD 21218, USA \\ Received 2018 October 18; revised 2018 November 8; accepted 2018 November 9; published 2019 January 3
}

\begin{abstract}
The bRing robotic observatory network was built to search for circumplanetary material within the transiting Hill sphere of the exoplanet $\beta$ Pic $\mathrm{b}$ across its bright host $\operatorname{star} \beta$ Pic. During the bRing survey of $\beta$ Pic, it simultaneously monitored the brightnesses of thousands of bright stars in the southern sky $\left(V \simeq 4-8, \delta \lesssim-30^{\circ}\right)$. In this work, we announce the discovery of $\delta$ Scuti pulsations in the A-type star HD 156623 using bRing data. HD 156623 is notable as it is a well-studied young star with a dusty and gas-rich debris disk, previously detected using ALMA. We present the observational results on the pulsation periods and amplitudes for HD 156623, discuss its evolutionary status, and provide further constraints on its nature and age. We find strong evidence of frequency regularity and grouping. We do not find evidence of frequency, amplitude, or phase modulation for any of the frequencies over the course of the observations. We show that HD 156623 is consistent with other hot and highfrequency pre-main sequence and early zero-age main sequence (ZAMS) $\delta$ Scutis as predicted by theoretical models and corresponding evolutionary tracks, although we observe that HD 156623 lies hotter than the theoretical blue edge of the classical instability strip. This, coupled with our characterization and Sco-Cen membership analyses, suggests that the star is most likely an outlying ZAMS member of the $\sim 16$ Myr Upper Centaurus-Lupus subgroup of the Sco-Cen association.
\end{abstract}

Key words: open clusters and associations: individual (Upper Centaurus-Lupus, Sco-Cen) - stars: early-type - stars: individual (HD 156623) - stars: oscillations - stars: pre-main sequence - stars: variables: delta Scuti

\section{Introduction}

The number of ground-based, wide-field photometric surveys that have been designed to detect transiting exoplanets has grown considerably over the past two decades (e.g., Bakos et al. 2002; Pollacco et al. 2006; Pepper et al. 2007; Talens et al. 2017). In addition to exoplanets, these surveys are sensitive to photometric variability due to eclipsing binaries, rotation periods, pulsations, among others (e.g., Mamajek et al. 2012; Mellon et al. 2017; Oberst et al. 2017; Burggraaff et al. 2018). These discoveries have been possible due to the highcadence, high-precision photometry of stars typically fainter than $V \simeq 7$. Fainter stars have been targeted due to their greater abundance per frame in the sky, making these surveys highly efficient.

These observations require exposure times on the order of tens of seconds to minutes. Brighter stars have been typically excluded from these surveys due to saturation of the detectors in their exposures. The bRing ( $\beta$ Pictoris b Ring) survey (Stuik et al. 2017) is a ground-based, wide-field photometric survey designed to monitor bright stars $(V \simeq 4-8)$, including the bright nearby exoplanet and debris disk host star $\beta$ Pic, and observes stars brighter than do many of the ground-based exoplanet surveys. Due to its high cadence and photometric precision, bRing is able to detect sub-mmag signals for these bright stars and is sensitive to large exoplanets and faint stellar signals, including $\delta$ Scuti pulsations. This paper presents the first science result from the bRing survey.

$\delta$ Scuti variable stars have been known to exhibit surface radial and non-radial pressure and gravitational pulsation modes (e.g., Fitch 1981; Balona \& Evers 1999; Breger 2000; Guenther et al. 2009; Zwintz et al. 2014a). One goal of asteroseismologists has been to develop models to identify and characterize these pulsations; such models can be used to probe the structure of a star (e.g., Kurtz et al. 2014) and help characterize its age and evolutionary status (e.g., Guenther et al. 2009; Zwintz et al. 2014b). These pulsation frequencies are also known to exhibit regular spacing in frequency and appear in groups (e.g., Zwintz et al. 2011, 2014a; Kurtz et al. 2014). $\delta$ Scutis have the tendency to lie within the classical instability strip on the Hertzsprung-Russell (H-R) diagram (Breger \& Pamyatnykh 1998), with some known to lie outside the strip (Bowman \& Kurtz 2018).

HD 156623 (HIP 84881, 2MASS J17205061-4525149) is a bright $(V=7.25$; Cousins \& Stoy 1962; Mermilliod 1997) A1 V star (Paunzen et al. 2001). Since 1962, it has served as a Harvard E region photometric standard in several systems, starting with the UBV system (Cousins \& Stoy 1962; Menzies et al. 1980, 1989; Cousins 1983) and later the 
$V R_{c} I_{c}$ (Cousins 1976, 1980; Menzies et al. 1980, 1989), uvby (Cousins 1987; Kilkenny \& Laing 1992), and $\mathrm{H} \beta$ systems (Cousins 1990). Reported mean photometry on the uvby $\beta$ system is provided in the compendia of Hauck \& Mermilliod (1997) and Paunzen (2015), and on the $U B V$ system by Mermilliod (1997).

The star was included both as a photometric standard and as being an A-type star in the vicinity of the Sco-Cen OB association by Slawson et al. (1992) (although no membership analysis was conducted). The star was first noted as having an infrared (IR) excess in the WISE survey by McDonald et al. (2012). Rizzuto et al. (2012) considered the star (listed as HIP 84881 ) as a low-probability member of Sco-Cen. The star's membership to Sco-Cen is not obvious as it lies well below the Galactic plane $\left(b=-4^{\circ} .8\right)$ on the opposite side from the Upper Centaurus-Lupus (UCL) subgroup of Sco-Cen (spanning $0^{\circ}<b<25^{\circ}$; de Zeeuw et al. 1999). Trigonometric parallaxes of $\varpi=8.45 \pm 0.60$ mas (van Leeuwen 2007, using Hipparcos astrometry) and $\varpi=8.9484 \pm 0.0769$ mas (Gaia Collaboration et al. 2016; Brown et al. 2018) have been reported, consistent with a distance $d=111.41_{-0.95}^{+0.97}$ pc (Bailer-Jones et al. 2018). Lieman-Sifry et al. (2016) report ALMA observations of HD 156623 , both $1240 \mu \mathrm{m}$ continuum and ${ }^{12} \mathrm{CO}(2-1)$ emission. Although detection of gas among young debris disk stars was relatively low in Sco-Cen $(\sim 16 \%)$ (Lieman-Sifry et al. 2016), ALMA surveys focusing specifically on young A-type stars are now showing that $\mathrm{CO}$ gas appears to be very common (Kral et al. 2017; Moór et al. 2017).

The work presented in this paper reports bRing's detection of $\delta$ Scuti pulsation frequencies in the A-type star HD 156623 and provides a characteristic analysis of the star and these frequencies. The paper is organized as follows: Section 2 describes the bRing observations; Section 3 details the data selection, reduction, frequency extraction, and stellar characterization; Section 4 discusses the refined properties of HD 156623 , reassessing its membership to Sco-Cen and estimated age, and the reported $\delta$ Scuti pulsation frequencies and their analysis.

\section{Observations}

\section{1. bRing Photometry}

The bRing survey was designed to monitor the 2017-2018 transit of the Hill sphere of the young giant exoplanet $\beta$ Pic b in front of the bright ( $V=3.8)$ star $\beta$ Pic, and search for evidence of any circumplanetary matter (Wang et al. 2016; Stuik et al. 2017; Mellon et al. 2018). One telescope (constructed at Leiden University) was installed in Sutherland, South Africa-an observing facility of the South African Astronomical Observatory in Cape Town-and the other (constructed at the University of Rochester) at Siding Spring Observatory in Coonabarabran, NSW, Australia. The South African bRing observatory has been observing continuously since 2017 January. The Australian bRing observatory started in 2017 November.

Each bRing station has two FLI Microline ML11002M cameras that were oriented to maximize the longitudinal coverage of $\beta \mathrm{Pic}$, which is nearly circumpolar at both sites (latitudes $\simeq-32^{\circ}$ ). One camera was pointed southeast $\left(\mathrm{Az}=150^{\circ}\right)$ and the other southwest $\left(\mathrm{Az}=210^{\circ}\right)$. The cameras came equipped with $4008 \times 2672$ pixel Grade 2 CCDs, which have a quantum efficiency (QE) of $\sim 50 \%$ and
$9 \mu \mathrm{m}$ pixels. The cameras were attached to Canon $24 \mathrm{~mm} f / 1.4$ wide-field lenses; this resulted in a $74^{\circ} \times 53^{\circ}$ field of view with $\sim 1^{\prime}$ pixels. The data were taken with alternating exposures of 6.38 and $2.54 \mathrm{~s}$ (Stuik et al. 2017).

The bRing calibration pipeline builds on the heritage of the MASCARA data pipeline (Stuik et al. 2014; Talens et al. 2017, 2018). The bRing pipeline (discussed in Stuik et al. 2017; Talens et al. 2018) utilizes relative aperture photometry calibrated to the ASCC catalog (Kharchenko 2001), which itself is tied to the $B V$ photometry of the Hipparcos and Tycho instruments (ESA 1997). The pipeline performs a preliminary data reduction correcting $\mathrm{CCD} \mathrm{QE}$, total throughput of the lenses and sky, intra-pixel variations, and sky- and cloud-based temporal variations. An astrometric solution is calculated every 50 exposures. For each camera, the resulting data are binned to a five minute cadence and updated for download on a biweekly basis.

In addition to $\beta$ Pic, the survey also monitored the brightnesses of $\sim 20,000$ of the brightest stars in the southern sky ( $V \simeq 4-8$ ), granting it a unique role among groundbased, wide-field photometric surveys (similar to its sibling MASCARA; Snellen et al. 2012, 2013; Stuik et al. 2014; Talens et al. 2017, 2018). The combined sky coverage of both sites has provided nearly continuous coverage of stars (an ideal $24 \mathrm{hr}$ day provides $20-21 \mathrm{hr}$ of continuous coverage). This nearly continuous temporal coverage combined with a cadence of five minutes has made bRing sensitive to serendipitous aperiodic and high-frequency, low-amplitude periodic events. The observations of HD 156623 in this work cover 2017 June-2018 May.

\subsection{FEROS Spectrum}

A FEROS spectrum of HD 156623 taken from the European Southern Observatory (ESO) archive was used in this analysis. The FEROS instrument is a high-resolution $(R=48,000)$ Échelle spectrograph located at the ESO in La Silla, Chile (Kaufer et al. 1999). Science products processed through the FEROS Data Reduction System are available for query and download on the ESO data archive. ${ }^{11}$

Although HD 156623 has been observed by FEROS several times at various exposure times, we analyzed a single spectrum with the highest signal-to-noise ratio (S/N) (ADP.2016 September 28T11:26:17.118; Rebollido et al. 2018). This observation has the longest exposure time (1800.04 s) and the highest $\mathrm{S} / \mathrm{N}$ (399.0). For the purposes of this work, this single spectrum was sufficient for providing estimates on the radial velocity and $v \sin i$.

\section{Analysis}

\subsection{Data Sample, Reduction, and Noise Discussion}

A subset of the bRing light curves was originally chosen to search for new variable stars. Stars were chosen if they had no previously reported variability (simply assessed via lacking an entry in the AAVSO VSX catalog; Watson et al. 2006) ${ }^{12}$ and no bright $(V \lesssim 10)$ neighboring stars within a $10^{\prime}$ radius (corresponding to four bRing inner apertures). These

\footnotetext{
11 http://archive.eso.org/wdb/wdb/adp/phase3_spectral/form?collection_ name $=$ FEROS

${ }^{12}$ The VSX catalog is regularly updated at http://cdsarc.u-strasbg.fr/viz-bin/ Cat?B/vsx.
} 
measurements were also used to help identify major systematics still present in the calibrated data.

Beyond the calibration, this study detrended additional strong systematics identified in the data. Due to the high cadence and stationary nature of bRing, strong systematic signals with frequencies at one sidereal day and one synodic day were generated from each star traveling across the CCD, as well as a systematic signal due to the Moon (Stuik et al. 2017; Burggraaff et al. 2018; Talens et al. 2018). These three sets of periodicities were simultaneously fit and detrended using an iterative median-binning routine. A second-order CCD QE response systematic was also fitted and subtracted for each camera. A heliocentric correction to the time series was also applied. Finally, the data from the individual cameras were median aligned to each other to remove scaling offsets due to the $\sim 1 \%$ differences in the calibrations. Composite light curves containing data from all four cameras were then generated. The detrended rms in each camera was also calculated for comparison to the predicted theoretical noise in each star.

At the time of this publication, the principal theoretical sources of Poisson noise have been quantified to first order, i.e., shot, background, dark current, and read noise. The uniqueness of the bRing survey and the detrended rms measurements of each star imply additional higher-order considerations must be included such as non-uniform transmission. We are currently working to incorporate these sources to better understand the residual noise and improve our post-calibration detrending routine. The goals of these analyses are to provide a postcalibration detrending routine that minimizes the residual noise in each star and to determine an overall scintillation noise-floor for each camera.

\subsection{Lomb-Scargle Analysis and Frequency Extraction}

The Lomb-Scargle (LS) periodogram (Scargle 1982; Press et al. 1992) is useful for detecting periodic signals in unevenly sampled data (e.g., Hartman et al. 2008, 2010; Messina et al. 2010; Cargile et al. 2014; Mellon et al. 2017). The LS periodogram function available in the Python Scipy ${ }^{13}$ package was used to verify the removal of the major systematics and to search for stars in this sample that show signs of previously undetected variability. For the periodograms in this work, the Nyquist frequency (Press et al. 1992) of $\sim 135$ day $^{-1}$ was used as an upper limit on the frequency with frequency spacings of $\sim 5 \times 10^{-4}$ day $^{-1}$. The Scipy normalized periodograms were generated for the composite light curve and four camera light curves.

The major systematics (sidereal, synodic, lunar) were clearly filtered out by our process with their peaks being cut by a factor of $\sim 100$. There were a few remaining low-frequency $\left(<5\right.$ day $\left.^{-1}\right)$ peaks that were only a few tenths of mmag above the residual noise level. These could be residual beats, interference frequencies, or unidentified systematics in bRing. Other than one possible systematic at $\sim 24$ day $^{-1}$ shared by some of the stars in this sample, there were no other systematic peaks in the periodograms of these stars. To check for new variables, these stars were studied in order of largest peak in the composite light curve periodogram until stars whose peaks were consistent with the noise level were found. This final sample of stars with peaks above the noise in their periodograms were phase-folded on their strongest periods and

\footnotetext{
${ }^{13}$ https://scipy.org/
}

Table 1

Adopted Observational Values for HD 156623

\begin{tabular}{lccc}
\hline \hline $\begin{array}{l}\text { Parameter } \\
(1)\end{array}$ & $\begin{array}{c}\text { Value } \\
(2)\end{array}$ & $\begin{array}{c}\text { Units } \\
(3)\end{array}$ & $\begin{array}{c}\text { Reference } \\
(4)\end{array}$ \\
\hline$\alpha$ & $260.21081592^{\mathrm{a}}( \pm 0.05 \mathrm{mas})$ & $\mathrm{deg}$ & 1 \\
$\delta$ & $-45.42100519^{\mathrm{a}}( \pm 0.06 \mathrm{mas})$ & $\mathrm{deg}$ & 1 \\
$\varpi$ & $8.9484 \pm 0.0769$ & $\mathrm{mas}$ & 1 \\
$d$ & $111.41_{-0.95}^{+0.97}$ & $\mathrm{pc}$ & 2 \\
$\mu_{\alpha}$ & $-14.3 \pm 0.1$ & $\mathrm{mas} \mathrm{yr}^{-1}$ & 1 \\
$\mu_{\delta}$ & $-40.1 \pm 0.1$ & $\mathrm{mas} \mathrm{yr}^{-1}$ & 1 \\
SpT & $\mathrm{A} 1 \mathrm{~V}$ & $\ldots$ & 3 \\
$V$ & $7.254 \pm 0.007$ & $\mathrm{mag}$ & 4 \\
$B-V$ & $0.087 \pm 0.003$ & $\mathrm{mag}$ & 4 \\
$(b-y)$ & $0.040 \pm 0.003$ & $\mathrm{mag}$ & 5 \\
$m_{1}$ & $0.193 \pm 0.004$ & $\mathrm{mag}$ & 5 \\
$c_{1}$ & $0.960 \pm 0.002$ & $\mathrm{mag}$ & 5 \\
$\beta$ & $2.904 \pm 0.001$ & $\mathrm{mag}$ & 5 \\
$E(B-V)$ & $0.008 \pm 0.018$ & $\mathrm{mag}$ & 6 \\
\hline
\end{tabular}

Notes. (1) Brown et al. (2018), (2) Bailer-Jones et al. (2018), (3) Paunzen et al. (2001), (4) Mermilliod (1997), (5) Paunzen (2015), (6) STILISM (Lallement et al. 2018)

${ }^{\text {a }}$ Epoch J2015.5, ICRS.

analyzed further to see if new variability or systematics were detected, i.e., the detected variability was either exclusive to a star or shared with other stars.

This analysis yielded evidence of $\delta$ Scuti pulsations for HD 156623, previously unrecognized as a variable likely due to its high-frequency $\left(f_{\text {detected }}>56\right.$ day $\left.^{-1}\right)$, low-amplitude $(<10 \mathrm{mmag})$ pulsations. All four bRing cameras had reported a total of $\sim 12,500$ data points between 2017 June 1 and the download date of 2018 May 28. The tool used to extract the frequencies was the literature standard Period04 software (Lenz \& Breger 2005). The Period0 4 analysis was performed on the composite light curve and the four individual camera light curves of HD 156623. The pre-whitening residual noise in the Fourier spectrum from Period04 for the composite light curve was $\sim 0.2 \mathrm{mmag}$. Periods were searched for down to $\mathrm{S} / \mathrm{N}=4$ (Kuschnig et al. 1997), which corresponded well with visual inspection of the Fourier spectrum revealing no more significant periods. The detected frequencies were entered into a spreadsheet for selection, analysis, and discussion (Section 4.2).

\subsection{Stellar Characteristics}

In preparation for the intended $\delta$ Scuti frequency analysis, we performed a stellar characterization analysis of HD 156623 to better constrain its properties. The adopted observables are summarized in Table 1, including Strömgren-Crawford (uvby $\beta$ ) photometry.

The Strömgren-Crawford photometric system is comprised of intermediate-band filters on either side of the Balmer break and two narrow-band filters that measure the strength of the $\mathrm{H} \beta$ line (Crawford 1958). For early-type stars, the strength of the Balmer break, the continuum slopes in this region, and the strength of the $\mathrm{H} \beta$ line are indicators of temperature and gravity. More specifically, for early A-type stars such as HD 156623 , the temperature is sensitive to the index $a_{0}$ :

$$
a_{0}=1.36(b-y)_{0}+0.36 m_{0}+0.18 c_{0}-0.2448
$$


Table 2

Derived Photometric Parameters for HD 156623

\begin{tabular}{lcc}
\hline \hline $\begin{array}{l}\text { Parameter } \\
(1)\end{array}$ & $\begin{array}{c}\text { Value (mag) } \\
(2)\end{array}$ & $\begin{array}{c}\text { Reference } \\
(3)\end{array}$ \\
\hline$A_{V}$ & $0.025 \pm 0.055$ & 1 \\
$E(b-y)$ & $0.0131 \pm 0.0092$ & 2 \\
$E\left(m_{1}\right)$ & $-0.0042 \pm 0.0029$ & 2 \\
$E\left(c_{1}\right)$ & $0.0026 \pm 0.0018$ & 2 \\
$E(u-b)$ & $0.020 \pm 0.014$ & 2 \\
$(b-y)_{0}$ & $0.0269 \pm 0.0096$ & 2 \\
$m_{0}$ & $0.1972 \pm 0.0050$ & 2 \\
$c_{0}$ & $0.9574 \pm 0.0027$ & 2 \\
$a_{0}$ & $0.035 \pm 0.013$ & 2 \\
$r^{*}$ & $-0.0058 \pm 0.0012$ & 2 \\
\hline
\end{tabular}

Note. (1) This paper, calculated using STILISM $E(B-V)$ value and $R_{V}$ from McCall (2004), (2) this paper, calculated using Fitzpatrick (1999).

while the surface gravity is highly sensitive to the reddeningfree index $r_{*}$ :

$$
r_{*}=0.35 c_{1}-0.07(b-y)-(\beta-2.565)
$$

where $m_{0}=(v-b)_{0}-(b-y)_{0} \quad$ and $\quad c_{0}=(u-v)_{0}-(v-b)_{0}$ (Napiwotzki et al. 1993).

For dereddening the photometry and estimating the extinction due to interstellar dust, we adopt the reddening based on the regularly updated tri-dimensional reddening maps from the STILISM program (Capitanio et al. 2017; Lallement et al. $2018)^{14}: E(B-V)=0.008 \pm 0.018$. Examining the results of McCall (2004) for low reddening and dwarf stars in the color range $-0.32<(B-V)_{o}<1.5$, one finds that the trend $A_{V} / E(B-V) \simeq R_{V}^{00}+0.167(B-V)_{o}$ is a reasonable approximation (where $R_{V}^{00} \simeq 3.07$ is quoted as the most probable value for a diffuse Galactic interstellar medium). Hence, we adopt the ratio of total to selective extinction $A_{V} / E(B-V)$ for our A1V star to be 3.08, and estimate the extinction to be $A_{V}=0.025 \pm 0.055$. Using the $E(B-V)$ value, we use the "observed" $u v b y \beta$ extinction ratios reported in Table 2 of Fitzpatrick (1999) to calculate the color excesses among the Strömgren-Crawford colors (see Table 2). The color excesses are all negligible, within $2 \sigma$ of zero.

We then followed a procedure similar to that of David \& Hillenbrand (2015) and derived $T_{\text {eff }}$ and $\log g$ through a twodimensional linear interpolation in the $a_{0}-r^{*}$ plane using a synthetic color grid distributed by Fiorella Castelli. ${ }^{15}$ The synthetic color grids were derived from ATLAS9 model atmospheres (Castelli \& Kurucz 2004, 2006). We calculated uncertainties in the atmospheric parameters using Monte Carlo simulations, modeling the input photometry as normal distributions with widths equal to the errors reported in Paunzen (2015) and using the previously estimated $A_{V}$ extinction value. The dereddened $u v b y \beta$ photometry and indices are presented in Table 2 and the position of HD 156623 in the $a_{0}-r^{*}$ plane with respect to the models is shown in Figure 1.

From this analysis we estimate $T_{\text {eff }}=9040_{-160}^{+240} \mathrm{~K}$ and $\log g=4.203_{-0.011}^{+0.018}$ dex. The values quoted here are the medians from the distributions resulting from the Monte Carlo

\footnotetext{
${ }^{14}$ https://stilism.obspm.fr/

15 http://wwwuser.oats.inaf.it/castelli/colors/uvbybeta/

uvbybetap00k2odfnew.dat
}

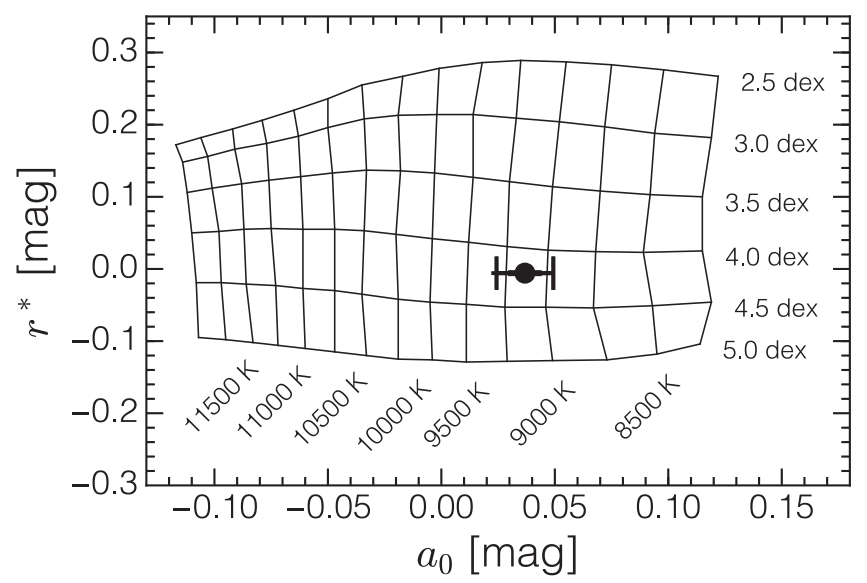

Figure 1. Position of HD 156623 (black circle) in the $a_{0}-r^{*}$ plane with respect to synthetic atmosphere colors (grid lines) from Castelli \& Kurucz $(2004,2006)$

simulations, while the errors quoted are determined from the 16th and 84th percentiles of those distributions. We note that these errors are statistical in nature, and we have not attempted to account for any systematic uncertainties intrinsic to the synthetic color grids. We chose instead to adopt a systematic uncertainty of 0.14 dex for $\log g$ based on the analysis from David \& Hillenbrand (2015). We adopted $T_{\text {eff }}=9040_{-160}^{+240} \mathrm{~K}$ and $\log g=4.20 \pm 0.14$ dex.

We calculated the luminosity and radius of HD 156623 using the photometrically derived parameters and Gaia parallax. We estimated the $V$-band bolometric correction using our estimated $T_{\text {eff }}$ combined with various published $B C_{V}$ tables, all scaled to the new IAU 2015 bolometric magnitude system. ${ }^{16}$ Here we adopt the solar apparent $V$ magnitude from Torres (2010) $(-26.76 \pm 0.03 \mathrm{mag})$ which, when combined with the IAU 2015 apparent bolometric magnitude $(-26.832 \mathrm{mag})$, equates to a solar $B C_{V}=-0.072 \pm 0.03 \mathrm{mag}$. Comparing the $B C_{V}$ scales from several studies (Balona 1994; Flower 1996; Bessell et al. 1998; Bertone et al. 2004), adjusting the scales slightly to reproduce $B C_{V}(5772 \mathrm{~K})=-0.072 \mathrm{mag}$, and accounting for the $T_{\text {eff }}$ uncertainties, we estimate the $V$-band bolometric correction for HD 156623 to be $-0.09 \pm 0.05$ mag. Accounting for the extinction $\left(A_{V}=0.025 \pm 0.055 \mathrm{mag}\right)$, we derive bolometric flux $f_{\text {bol }}=35.11 \pm 2.42 \mathrm{pW} \mathrm{m}^{-2}$, apparent bolometric magnitude $m_{\mathrm{bol}}=7.139 \pm 0.075 \mathrm{mag}$, absolute bolometric magnitude $M_{\text {bol }}=1.898 \pm 0.077 \mathrm{mag}$ (IAU 2015 system), and absolute magnitude $M_{V}=1.988 \pm 0.058 \mathrm{mag}$. We derive bolometric luminosity $\log \left(L / L_{\odot}\right)=1.137 \pm 0.031 \mathrm{dex}$ or $13.71_{-0.94}^{+1.01} L_{\odot}$, on the IAU 2015 scale where $L_{\odot}=3.828 \times 10^{26} \mathrm{~W}$. Combined with the previously estimated effective temperature $\left(T_{\text {eff }}=\right.$ $9040_{-160}^{+240} \mathrm{~K}$ ), we estimate the stellar radius to be $1.51 \pm 0.09 R_{\odot}$, where $R_{\odot}$ is the IAU 2015 nominal solar radius of $695,700 \mathrm{~km}$. These estimated stellar parameters are summarized in Table 3.

To provide a consistency check on the parameters derived for HD 156623, we used the isoclassify code from Huber et al. (2017), which uses the MIST evolutionary models (Choi et al. 2016; Dotter 2016) as well as the 3D reddening map of Green et al. (2015) implemented in the mwdust package

\footnotetext{
${ }^{16}$ https://www.iau.org/static/resolutions/IAU2015_English.pdf; see also Mamajek et al. (2015). The IAU 2015 bolometric magnitude system is set so that $M_{\mathrm{bol}}=4.74 \mathrm{mag}$ corresponds to the nominal solar luminosity of $3.828 \times 10^{26} \mathrm{~W}$.
} 
Table 3

Parameters Derived for HD 156623

\begin{tabular}{lcc}
\hline \hline $\begin{array}{l}\text { Parameter } \\
(1)\end{array}$ & $\begin{array}{c}\text { Value } \\
(2)\end{array}$ & $\begin{array}{c}\text { Units } \\
(3)\end{array}$ \\
\hline$T_{\text {eff }}$ & $9040_{-160}^{+240}$ & $\mathrm{~K}$ \\
$\log g$ & $4.20 \pm 0.14$ & $\mathrm{dex}$ \\
$\mathrm{BC}_{V}$ & $-0.09 \pm 0.05$ & $\mathrm{mag}$ \\
$M_{V}$ & $1.99 \pm 0.06$ & $\mathrm{mag}$ \\
$M_{\text {bol }}$ & $1.90 \pm 0.08$ & $\mathrm{mag}$ \\
$\log \left(L / L_{\odot}\right)$ & $1.137 \pm 0.031$ & $\mathrm{dex}$ \\
Radius & $1.51 \pm 0.09$ & $R_{\odot}$ \\
Age & $16 \pm 7$ & $\mathrm{Myr}$ \\
$v_{r}$ & $3.8 \pm 6.9$ & $\mathrm{~km} \mathrm{~s}^{-1}$ \\
$v \sin i$ & $88 \pm 2$ & $\mathrm{~km} \mathrm{~s}^{-1}$ \\
\hline
\end{tabular}

(Bovy et al. 2016). The isoclassify code has two modes: direct and grid. In the direct mode, the code takes an input parallax, $K$-band magnitude and priors on $T_{\text {eff }}, \log g$, and $[\mathrm{Fe} / \mathrm{H}]$ in order to determine the luminosity and radius using theoretical $K$-band bolometric corrections. The parallax is used to generate a distance posterior distribution, which is then used to calculate reddening given the star's position and a 3D dust map. In the grid mode, the code does not rely on a reddening map but instead treats extinction as a free parameter. Using a fine grid of MIST isochrones, the code calculates reddened photometry in the 2MASS $J H K$, Tycho $B_{T} V_{T}$, and Sloan griz passbands for given values of $A_{V}$ by interpolating the Cardelli et al. (1989) extinction law. The code then integrates over all isochrone points to find the maximum likelihood model that matches various combinations of input observables (e.g., colors).

The $\log g$ derived from $u v b y \beta$ photometry is consistent within $1 \sigma$ of the value found using the isoclassify grid mode $(4.28 \pm 0.01 \mathrm{dex})$. The radii $\left(R_{\text {grid }}=1.635 \pm 0.026 R_{\odot}, R_{\text {direct }}\right.$ $\left.1.618 \pm 0.092 R_{\odot}\right), T_{\text {eff }}\left(T_{\text {eff,grid }}=8920 \pm 230 \mathrm{~K}, T_{\text {eff,direct }}=\right.$ $9040 \pm 240 \mathrm{~K}), \quad$ and luminosity $\quad\left(\log \left(L / L_{\odot, \text { grid }}\right)=1.18 \pm\right.$ $\left.0.04 \mathrm{dex}, \log \left(L / L_{\odot \text {,direct }}\right)=1.20 \pm 0.01 \mathrm{dex}\right)$ values found using both modes are consistent with one another and the results in Table 3 within $\sim 2 \sigma$.

\subsection{Spectral Analysis}

The FEROS spectrum of HD 156623 was downloaded in the FITS file format and analyzed with the SPLAT-VO tool. ${ }^{17}$ The wavelength range of $3900-6800 \AA$ was used to avoid the Balmer jump and near-IR telluric lines interfering with the normalization process. Within SPLAT-VO, the spectrum was normalized by carefully using the line draw tool to interpolate an estimated continuum. The normalized spectrum was then analyzed to determine several properties of the star.

The Balmer series, Ca II K, and several metal lines in the spectrum (summarized in Table 4) were fit in SPLAT-VO with Gaussian, Lorentzian, and Voigt profiles. The best profile for each individual line was determined by the lowest rms of their fit. The profiles returned central wavelengths for each line, which were used to estimate a radial velocity for the star. The central wavelengths were compared to air wavelengths available in the NIST Atomic Spectra database. ${ }^{18}$ Based on this analysis, the radial velocity for HD 156623 was determined

\footnotetext{
17 http://star-www.dur.ac.uk/ pdraper/splat/splat-vo/

18 http://physics.nist.gov/PhysRefData/ASD/lines_form.html
}

Table 4

Radial Velocity Spectral Lines

\begin{tabular}{lcc}
\hline \hline Element & Air Wavelength $(\AA)$ & Measured Wavelength $(\AA)$ \\
$(1)$ & $(2)$ & $6562.779 \pm 0.034$ \\
\hline $\mathrm{H} \alpha$ & 6562.800 & $4861.387 \pm 0.033$ \\
$\mathrm{H} \beta$ & 4861.330 & $4340.303 \pm 0.030$ \\
$\mathrm{H} \gamma$ & 4340.470 & $4101.946 \pm 0.025$ \\
$\mathrm{H} \delta$ & 4101.760 & $3933.654 \pm 0.050$ \\
$\mathrm{Ca}$ II K & 3933.636 & $4128.053 \pm 0.045$ \\
$\mathrm{Si}$ II & 4128.070 & $4215.577 \pm 0.015$ \\
$\mathrm{Sr}$ II & 4215.519 & $4226.882 \pm 0.011$ \\
$\mathrm{Ca}$ I & 4226.728 & $4233.273 \pm 0.017$ \\
$\mathrm{Fe}$ II & 4233.167 & $4246.921 \pm 0.013$ \\
$\mathrm{Sc}$ II & 4246.820 & $4250.509 \pm 0.011$ \\
$\mathrm{Fe}$ II & 4250.429 & $4271.638 \pm 0.012$ \\
$\mathrm{Fe}$ II & 4271.400 & $4289.962 \pm 0.011$ \\
$\mathrm{Fe}$ II & 4289.775 & $4404.717 \pm 0.014$ \\
$\mathrm{Fe}$ I & 4404.750 & $4416.319 \pm 0.053$ \\
$\mathrm{Fe}$ II & 4416.339 & $4468.456 \pm 0.022$ \\
$\mathrm{Ti}$ II & 4468.507 & \\
\hline
\end{tabular}

to be $v_{r}=3.8 \pm 6.9 \mathrm{~km} \mathrm{~s}^{-1}$, which is reported in Table 3 . The best value for the radial velocity was determined from the average value of the $v_{r}$ measured from each line; the uncertainty was propagated from the fit errors on the measured central wavelengths. Notably absent from this analysis were the $\mathrm{H} \epsilon$ and $\mathrm{Ca}$ II $\mathrm{H}$ lines and the $\mathrm{Mg}$ II doublet at $4481 \AA$. Both sets of lines were blended in this spectrum and led to radial velocity values that were incompatible with those measured in the other lines. This led to their exclusion from this analysis.

To estimate $v \sin i$ for HD 156623, several synthetic spectra from the POLLUX ${ }^{19}$ service were generated. The POLLUX (Palacios et al. 2010) service provides synthetic ATLAS12 (Kurucz 2005) atmospheres for early-A stars with $T_{\text {eff }}$ in steps of $100 \mathrm{~K}, \log g$ in steps of $0.1 \mathrm{dex}$, and $[\mathrm{Fe} / \mathrm{H}]$ in steps of 0.5 dex. Within the POLLUX service, the ATLAS12 models can be convolved with a synthetic broadening profile based on the work by Gray (2005). This broadening profile is determined by an input macroturbulent velocity, rotational velocity, instrument profile, and radial velocity. To extract a $v \sin i$, we chose to fit these artificially broadened ATLAS12 models from the POLLUX service to the well-normalized $\mathrm{H} \beta$ and narrow metal lines present in the spectrum. This method for estimating $v \sin i$ is similar to Method 2 described in Brown \& Verschueren (1997) and is applicable for $50 \mathrm{~km} \mathrm{~s}^{-1} \leqslant$ $v \sin i \leqslant 200 \mathrm{~km} \mathrm{~s}^{-1}$.

For a simple estimate of $v \sin i$, we assumed a broadening profile due to rotation only, i.e., $v_{\text {macro }}=0 \mathrm{~km} \mathrm{~s}^{-1}$ and negligible instrument profile. The lines used to determine $v \sin i$ were selected based on their strength in the HD 156623 spectrum and their use in previous studies $(\mathrm{H} \beta$, Fe II $4233 \AA$, Ca I $4226 \AA$, Mg I 4702 A; Ramella et al. 1989; Royer et al. 2002, 2014; Zwintz et al. 2014a). The first line analyzed was the Fe II $\AA$ line. This line provided a strong means of measuring a close value for the atmospheric parameters and broadening profile simultaneously. With initial guesses based on the Strömgren-derived parameters, we varied $T_{\text {eff }}, \log g,[\mathrm{Fe} / \mathrm{H}]$, and $v \sin i$ in steps of $100 \mathrm{~K}, 0.1 \mathrm{dex}, 0.5 \mathrm{dex}$, and $10 \mathrm{~km} \mathrm{~s}^{-1}$ respectively. Each fit was evaluated with a $\chi^{2}$ value. This

19 http://pollux.graal.univ-montp2.fr/ 


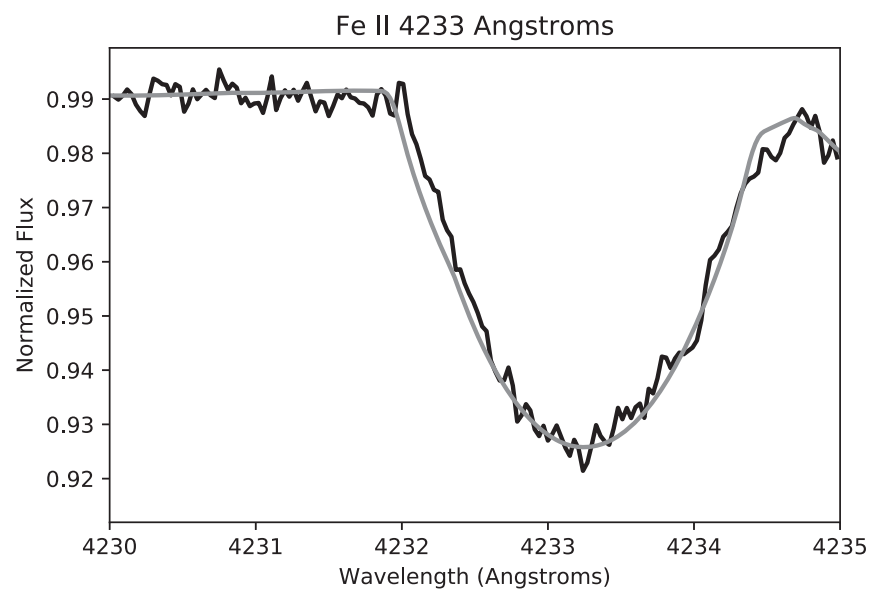

Figure 2. Example fit using the Fe II line at $4233 \AA$. The black line represents the normalized FEROS spectrum. The gray line is the best-fit artificially broadened ATLAS12 model spectrum $\left(T_{\text {eff }}=9200 \mathrm{~K}, \quad \log g=4.2 \mathrm{dex}, \quad[\mathrm{Fe} / \mathrm{H}]=\right.$ -0.5 , and $v \sin i=87 \mathrm{~km} \mathrm{~s}^{-1}$ ).

located a starting atmosphere of $T_{\text {eff }}=9200 \mathrm{~K}, \log g=$ $4.2 \mathrm{dex},[\mathrm{Fe} / \mathrm{H}]=-0.5 \mathrm{dex}$, and $v \sin i=90 \mathrm{~km} \mathrm{~s}^{-1}$.

This initial guess of $v \sin i=90 \mathrm{~km} \mathrm{~s}^{-1}$ was then varied in steps of $\pm 2 \mathrm{~km} \mathrm{~s}^{-1}$ and then $\pm 1 \mathrm{~km} \mathrm{~s}^{-1}$. This resulted in a best-fit $v \sin i$ for the Fe II line of $T_{\text {eff }}=9200 \mathrm{~K}, \log g 4.2$ dex, $[\mathrm{Fe} / \mathrm{H}]=-0.5 \mathrm{dex}$, and $v \sin i=87 \mathrm{~km} \mathrm{~s}^{-1}$. The Fe II $4233 \AA$ line and its best fit are plotted in Figure 2 as an example. The black line in Figure 2 represents the measured FEROS spectrum and the gray line represents the best-fit theoretical spectrum. The other two metal lines were best fit with this same atmosphere and the $\mathrm{H} \beta$ line was better fit with $T_{\text {eff }}=9300 \mathrm{~K}$ and $v \sin i=90 \mathrm{~km} \mathrm{~s}^{-1}$. Using these four lines, we adopt $v \sin i=88 \pm 2 \mathrm{~km} \mathrm{~s}^{-1}$. Our velocity results are consistent with Rebollido et al. (2018).

\section{Results and Discussion}

\subsection{Group Membership and Age}

The results and values in Table 3 were used to evaluate the age of HD 156623 based on its H-R diagram position and perform a Sco-Cen membership analysis. The H-R diagram position of the star is plotted (in Figure 3) along with solar composition isochrones from the PARSEC tracks (Marigo et al. 2017). ${ }^{20}$ The H-R diagram position for the star is consistent with a zero-age main sequence (ZAMS) star, likely older than $\sim 11 \mathrm{Myr}$ and younger than $\sim 200 \mathrm{Myr}$.

Applying the BANYAN $\Sigma$ kinematic membership tool ${ }^{21}$ (which assesses membership probabilities for stars based on position and kinematic data (Gagné et al. 2018)) with the Gaia DR2 astrometry, we found a membership probability of $88.6 \%$ for the HD 156623 to belong to the UCL subgroup of Sco-Cen (de Zeeuw et al. 1999; Mamajek et al. 2002; Preibisch \& Mamajek 2008; Pecaut \& Mamajek 2016). BANYAN $\Sigma$ also estimated a $11.4 \%$ probability that the star is a field star, and a probability of $<0.1 \%$ for it to belong to any of the other known kinematic groups within $150 \mathrm{pc}$ included in the code's database. If the star belongs to UCL, its predicted radial velocity is $3.2 \mathrm{~km} \mathrm{~s}^{-1}$, which is consistent with our measured

\footnotetext{
${ }^{20}$ Isochrones generated via the online CMD 3.0 tool at http://stev.oapd.inaf.it/ cgi-bin/cmd, using initial composition $X=0.7092, Y=0.2755, Z=0.0152$.

${ }^{21}$ http://www.exoplanetes.umontreal.ca/banyan/banyansigma.php
}

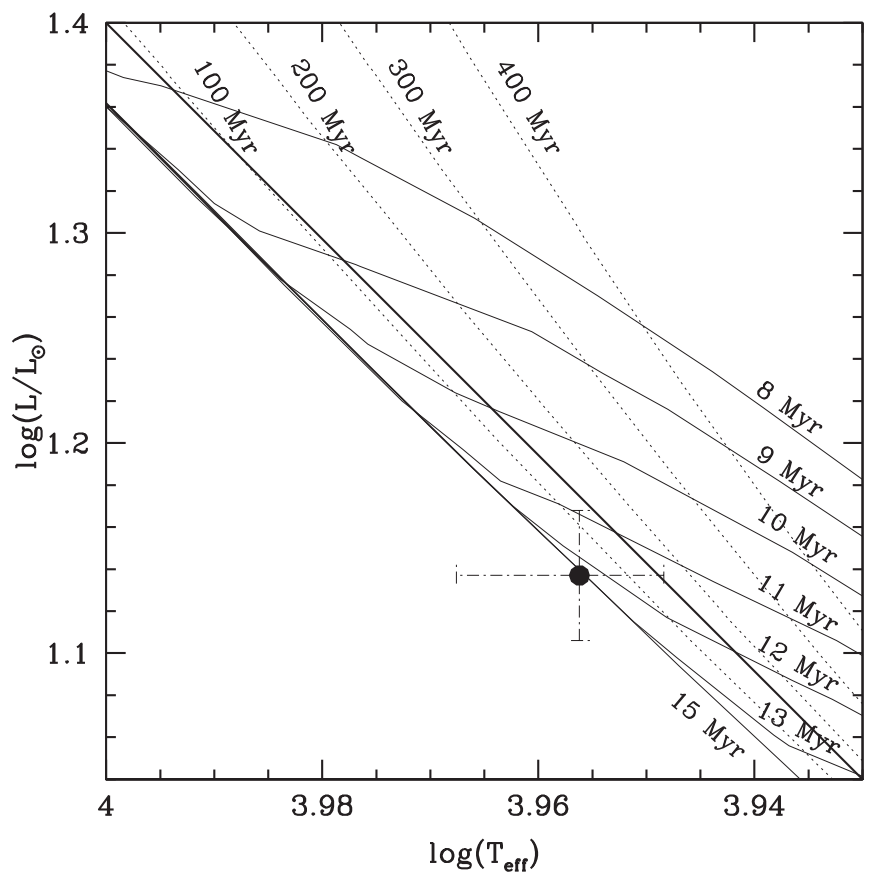

Figure 3. H-R diagram position of HD 156623 plotted along with PARSEC track solar composition isochrones. The position on the H-R diagram is consistent with a zero-age main sequence star between $\sim 11 \mathrm{Myr}$ and $\sim 200$ Myr.

value of $3.8 \pm 6.9 \mathrm{~km} \mathrm{~s}^{-1}$. UCL has a mean age of $16 \pm 2 \mathrm{Myr}$; however, the group covers tens of parsecs, and appears to have an intrinsic age spread of approximately \pm 7 Myr (Pecaut \& Mamajek 2016). UCL surrounds the Lupus clouds, with $\sim 5-10$ Myr-old stars in the vicinity (but outside of the star-forming clouds), and pockets of UCL appear to have ages as old $\sim 20-25 \mathrm{Myr}$. We surmise that HD 156623 is most likely an outlying member of UCL, with age $\sim 16 \pm 7 \mathrm{Myr}$, based on the intrinsic scatter in the ages of UCL members from Pecaut \& Mamajek (2016), and consistent with the H-R diagram position. This suggests that the star is ZAMS, or possibly slightly pre-main sequence (pre-MS) if it is younger than about $<12$ Myr.

\section{2. $\delta$ Scuti Frequencies}

\subsubsection{Reported Frequencies}

The analysis of Section 3.2 recovered 16 p-mode frequencies for HD 156623. Since the noise characteristics of bRing were not completely understood (see Section 3.1), this study was conservative when reporting frequencies as astrophysical. Due to the design of bRing, we had the ability to check for each frequency in each camera individually when selecting frequencies. These 16 frequencies were subsequently broken into a group of nine confirmed frequencies (Table 5) and seven candidate frequencies (Table 6). The nine confirmed frequencies were detected in data taken by at least one camera at each site. This criterion guaranteed that these frequencies were not single-camera and single-site systematics being mistakenly reported as real astrophysical frequencies intrinsic to the star. The seven candidate frequencies were only detected in the South African West Camera (SAW), which had observed the star the most with $\sim 8500$ data points. These frequencies reported in Table 6 could be either real astrophysical frequencies or unknown systematics intrinsic to either SAW 
Table 5

Nine Confirmed $\delta$ Scuti Frequencies

\begin{tabular}{lccc}
\hline \hline $\begin{array}{l}\text { Frequency } \\
\left(\text { day }^{-1}\right)\end{array}$ & $\begin{array}{c}\text { Amplitude } \\
(\mathrm{mmag})\end{array}$ & $\begin{array}{c}\text { Phase } \\
(\mathrm{Rad})\end{array}$ & $\begin{array}{c}\text { \# of Cameras } \\
-\end{array}$ \\
\hline $71.143 \pm 0.002$ & $6.63 \pm 1.23$ & $0.565 \pm 0.001$ & 4 \\
$67.005 \pm 0.002$ & $2.60 \pm 0.99$ & $0.542 \pm 0.001$ & 4 \\
$67.306 \pm 0.002$ & $2.31 \pm 0.65$ & $0.114 \pm 0.001$ & 4 \\
$63.562 \pm 0.002$ & $2.18 \pm 1.29$ & $0.570 \pm 0.001$ & 3 \\
$63.426 \pm 0.002$ & $1.70 \pm 0.34$ & $0.072 \pm 0.001$ & 4 \\
$59.002 \pm 0.003$ & $1.69 \pm 0.33$ & $0.413 \pm 0.001$ & 3 \\
$63.701 \pm 0.003$ & $1.58 \pm 0.31$ & $0.637 \pm 0.001$ & 3 \\
$59.970 \pm 0.003$ & $1.03 \pm 0.09$ & $0.556 \pm 0.001$ & 2 \\
$70.880 \pm 0.003$ & $1.00 \pm 0.18$ & $0.258 \pm 0.001$ & 2 \\
\hline
\end{tabular}

Note. Mean epoch 2458193.3 HJD.

Table 6

Seven Candidate $\delta$ Scuti Frequencies

\begin{tabular}{lcc}
\hline \hline $\begin{array}{l}\text { Frequency } \\
\left(\text { day }^{-1}\right)\end{array}$ & $\begin{array}{c}\text { Amplitude } \\
(\mathrm{mmag})\end{array}$ & $\begin{array}{c}\text { Phase } \\
(\text { Rad })\end{array}$ \\
\hline $93.261 \pm 0.002$ & $1.04 \pm 0.78$ & $0.896 \pm 0.001$ \\
$60.700 \pm 0.002$ & $0.95 \pm 0.78$ & $0.873 \pm 0.001$ \\
$75.211 \pm 0.002$ & $0.82 \pm 0.78$ & $0.882 \pm 0.002$ \\
$66.414 \pm 0.002$ & $0.79 \pm 0.78$ & $0.457 \pm 0.002$ \\
$64.179 \pm 0.002$ & $0.75 \pm 0.78$ & $0.581 \pm 0.002$ \\
$89.250 \pm 0.002$ & $0.73 \pm 0.78$ & $0.434 \pm 0.002$ \\
$56.116 \pm 0.002$ & $0.70 \pm 0.78$ & $0.198 \pm 0.002$ \\
\hline
\end{tabular}

Note. Mean epoch 2458193.3 HJD.

or the South African bRing station itself. We also include in Table 5 the number of cameras each frequency was detected in. The cameras that missed some of the frequencies were either the Australia West or South Africa East cameras, which both observed the star the least at their respective sites.

The frequencies and phases reported in Table 5 were those detected using Period04 on the composite periodograms. The reported amplitudes in Table 5 were from averaging the composite and individual camera detections uncovered by Period04. The uncertainties for the frequencies and amplitudes were calculated based on the measurements made in each camera and the composite light curve. This resulted in uncertainties that were one order of magnitude larger in frequency than those calculated using the methods of Montgomery \& Odonoghue (1999), but the presence of remaining unidentified systematics due to the high observing cadence and stationary nature of bRing requires a more conservative estimate of the noise until these systematics are investigated further and removed. The phase uncertainties, however, were calculated via Montgomery \& Odonoghue (1999) using the amplitudes from Table 5. The frequencies, amplitudes, and phases in Table 6 were reported as detected in SAW. The uncertainties for the frequency and amplitude were computed using those from Table 5. The uncertainty in the phase for Table 6 was similarly computed from Montgomery \& Odonoghue (1999). The amplitude uncertainties were rather large compared to the reported amplitudes. The culprit was likely slight temporal systematics between the cameras.

To ensure that the frequencies were exclusive to HD 156623, the three nearest neighbors in the bRing catalog (HD 157661, $\sim 68$ pixels apart, HD 156274, $\sim 75$ pixels apart, and HD $156293, \sim 80$ pixels apart) were used as check stars. They were
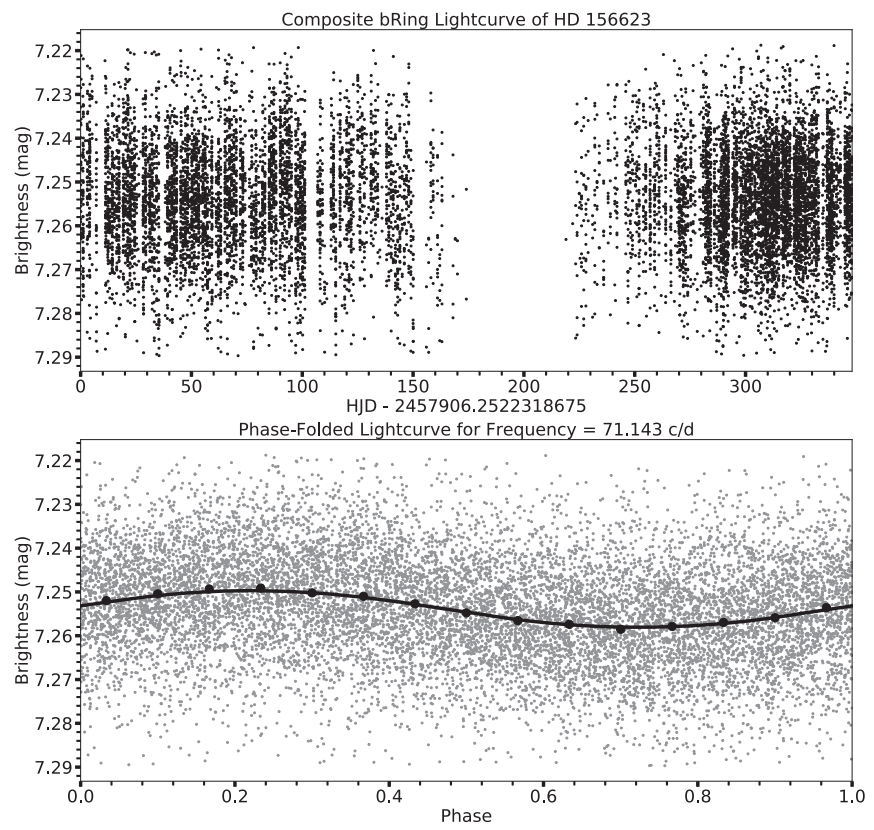

Figure 4. Top panel: composite bRing light curve of HD 156623. Bottom panel: composite bRing light curve (gray dots) phase-folded onto the primary 71.143 day $^{-1}$ frequency. The black circles are 15 median binned data points and the black curve is the sinusoidal solution found by Period0 4 .

put through the same data reduction and periodogram analysis discussed in Section 3. They were chosen as they should be subject to similar sources of global and local noise on the CCD due to their proximity. Therefore, any unique signals detected in the periodogram of HD 156623 should be independent of any signals detected in the periodograms of these stars. We found no evidence of pulsations within the 50-100 day ${ }^{-1}$ frequency range of these three check stars.

In the top panel of Figure 4, we plot the full composite light curve of HD 156623. In the bottom panel we plot a phasefolded light curve of the primary frequency $\left(71.143\right.$ day $\left.^{-1}\right)$. This bottom plot includes the bRing data in gray, 15 evenly spaced median-binned data points represented by black circles, and the sinusoidal solution from Period04 plotted as a black line.

\subsubsection{Interpreting the Frequencies}

HD 156623 has been a photometric standard star for decades. It is expected that any new detected pulsations in the star should be comparable or smaller than the best value of the $V$ magnitude and its associated uncertainty $(V=7.24 \pm$ 0.007 mag; Mermilliod et al. 1997). Considering the confirmed frequencies only, one finds the sum of the amplitudes to be $\sim 20 \mathrm{mmag}$, which is around three times lower than the standard error of the mean of the $V$ magnitude, hence it is unsurprising that the $\delta$ Scuti pulsations were previously missed.

In the top panel of Figure 5, the periodogram of the HD 156623 composite light curve is plotted between 56 and 93.5 day $^{-1}$, within which the $\delta$ Scuti pulsations are contained. The second panel shows the periodogram after removal of the primary frequency and the third shows the residual periodogram after the removal of all frequencies above the noise threshold. The projections of the nine reported frequencies from Table 5 are plotted with solid lines in the bottom panel of Figure 5 along with the projections of the seven candidate frequencies from Table 6 plotted with dashed lines. The main 


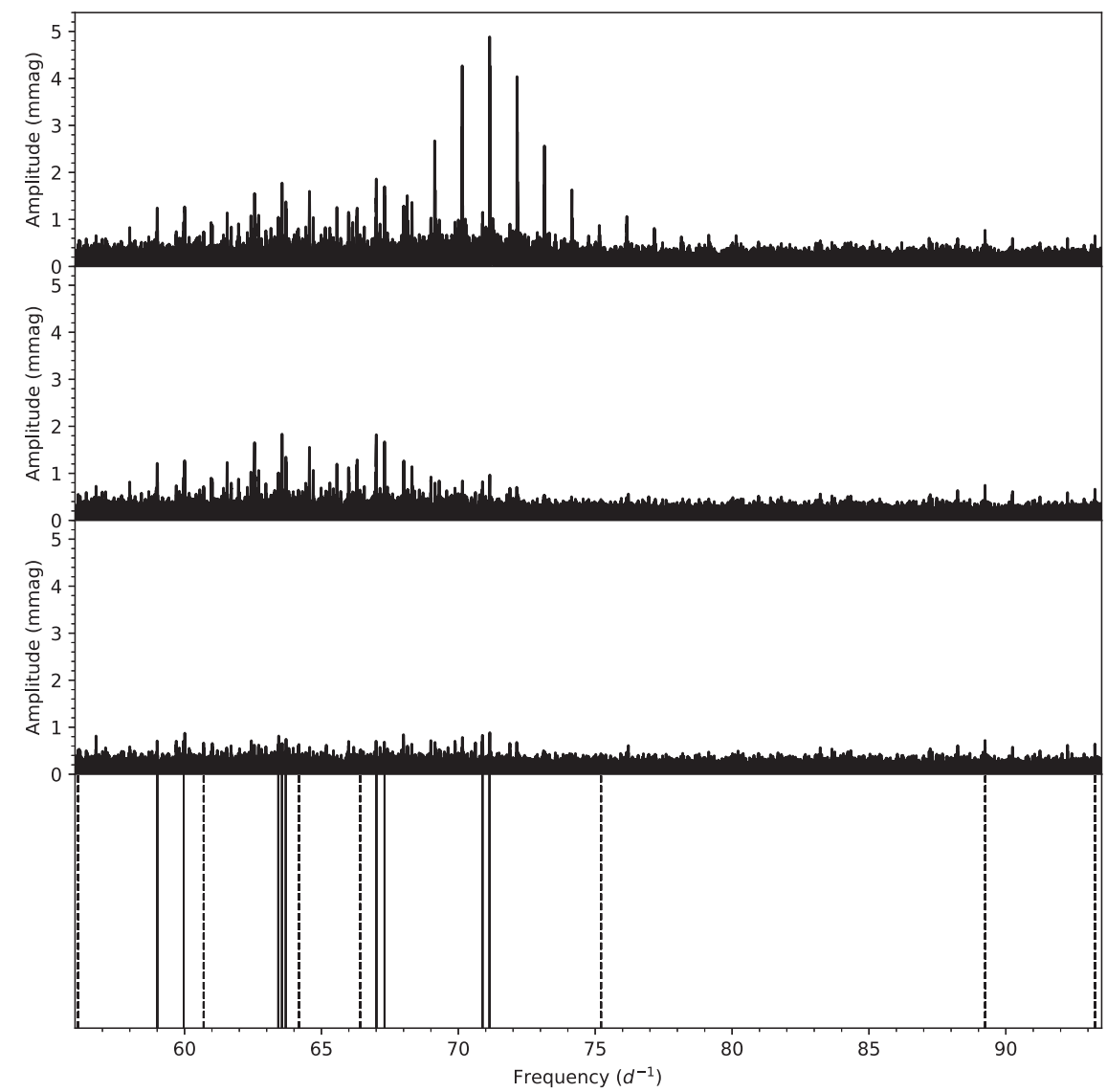

Figure 5. Lomb-Scargle periodogram of the HD 156623 composite light curve plotted in the top panel. The second panel shows the residual periodogram after the removal of the primary frequency. The third panel shows the residual noise periodogram. In the bottom panel, the projections of the nine confirmed frequencies from Table 5 are plotted with solid lines and the seven candidate frequencies from Table 6 are plotted with dashed lines. The frequency span is between 56 and 93.5 day ${ }^{-1}$ to focus on the $\delta$ Scuti features. It should be noted that the amplitudes plotted in the top panel periodogram correspond to the amplitudes from the periodogram of the composite light curve and not the reported amplitudes from Tables 5 and 6.

frequency $\left(71.143 \mathrm{day}^{-1}\right)$ generated several daily aliases, which are obvious in the periodogram. There were also several frequencies that seemed real; upon closer inspection, these appeared to be aliases between the peaks (several strong beat frequencies were also generated and are outside the window of Figure 5). The reported frequencies were not aliases of each other or linear combinations of one another. They were also well-resolved, despite our large uncertainties. In HD 156623, we clearly observed high-order radial modes (the fundamental radial should not exceed 25 day $^{-1}$; Zwintz et al. 2011).

There was evidence of grouping between all 16 frequencies (frequencies clustered around each other; Kurtz et al. 2014). Without a proper model and seven of our frequencies only reaching candidate status, we deduced a possible interpretation of how the frequencies may be grouped based on a visual inspection of Figure 5. The main frequency $\left(71.143 \mathrm{day}^{-1}\right)$ appeared to exist in a doublet with the frequency $70.880 \mathrm{day}^{-1}$. We also saw evidence for two sets of triplets (\{67.005 day ${ }^{-1}, 67.306 \mathrm{day}^{-1}$, 66.414 day $\left.^{-1}\right\}$ and $\left\{59.002\right.$ day $^{-1}, 59.970$ day $^{-1}, 60.700$ day $\left.\left.^{-1}\right\}\right)$ and one quadruplet $\left\{63.562\right.$ day $^{-1}, 63.426$ day $^{-1}, 63.701$ day $^{-1}$, 64.179 day $\left.^{-1}\right\}$. The rest of the frequencies appeared to be singlets. The frequencies from this analysis showed strong evidence of regularity (frequencies with common separation; Zwintz et al. 2011). We plot a histogram of the differences between all 16 frequencies-using bins of size $0.5 \mathrm{day}^{-1}$-in Figure 6; we find evidence of regularity for three different separations: $3.75,7.25$, and 2.75 day $^{-1}$. To generate an accurate

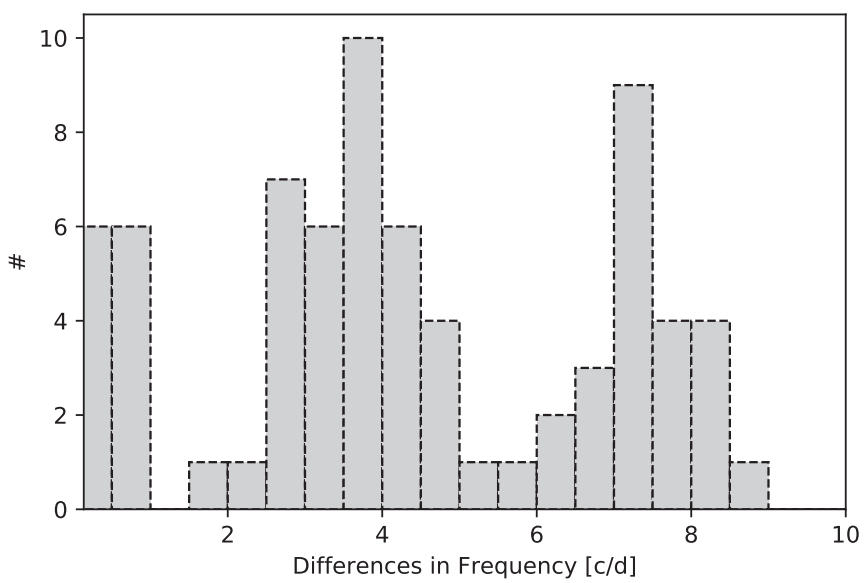

Figure 6. Histogram of frequency differences using all 16 frequencies detected by this study $\left(0.5 \mathrm{day}^{-1}\right.$ bins). This histogram shows strong evidence of regularity at the spacings of $3.75,7.25$, and 2.75 day $^{-1}$.

interpretation of these regularities and groupings, one would need to develop a model similar to that of Zwintz et al. (2014a), which is beyond the scope of this work.

To check for temporal variations in the frequencies, amplitudes, and phases, the data were broken up into roughly two month segments in SAW (the best camera to individually sample the star with $\sim 8500$ points). All three parameters for each set of frequencies stayed consistent (within uncertainty) 


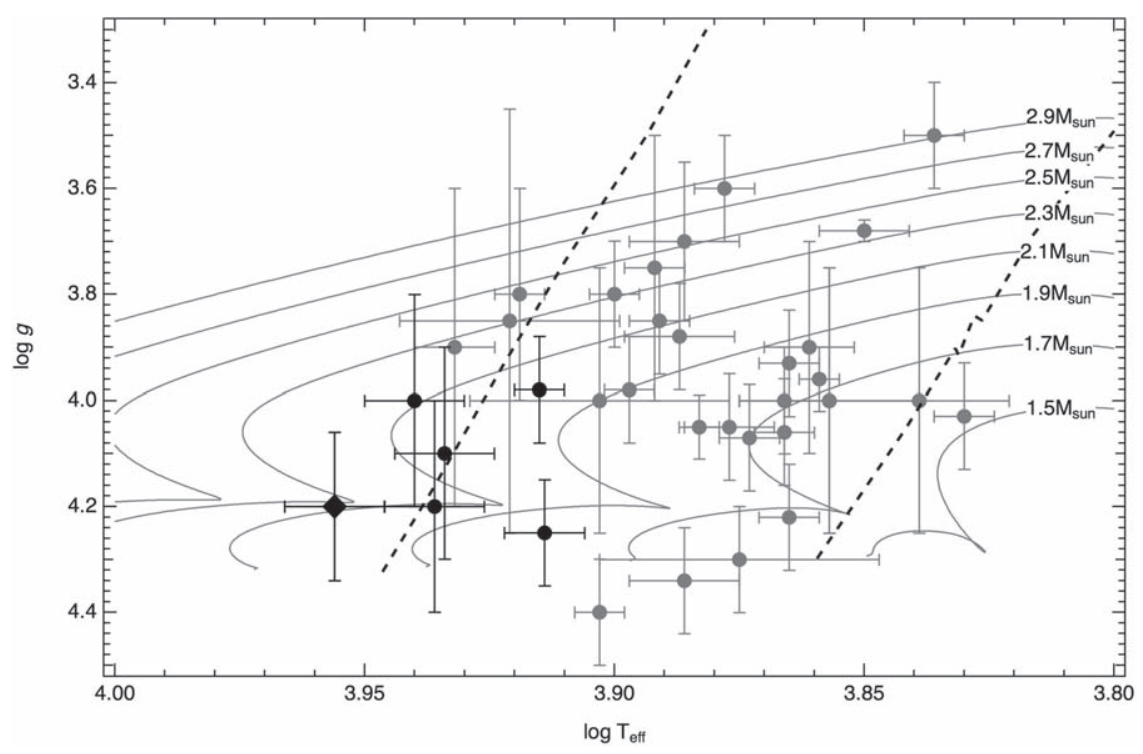

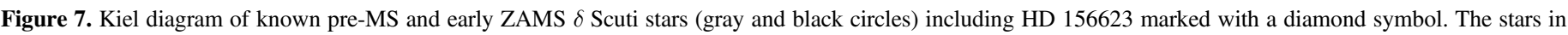

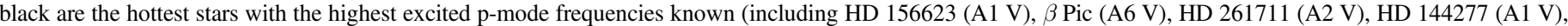

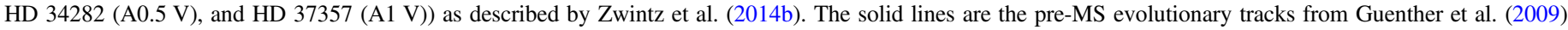

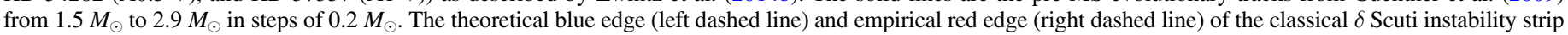
are also plotted (Breger \& Pamyatnykh 1998).

throughout each segment. Therefore, period, amplitude, and phase variations were not detected in this study on the timescale of around one year.

\subsubsection{Evolutionary Stage Analysis}

A critical parameter in the seismic analysis of pre-MS and ZAMS stars is the acoustic-cutoff frequency (Zwintz et al. $2014 b)$. This corresponds to the highest-frequency $\left(f_{\max }\right)$ pressure mode in the star (Zwintz et al. 2014a). Casey (2011) predicted that the highest acoustic-cutoff frequencies occur as the star approaches ZAMS. It is also expected that the acousticcutoff frequency scales with the frequency of maximum power (Aerts et al. 2010). The echographic study of Zwintz et al. (2014b) used pre-MS and ZAMS $\delta$ Scutis to show that the hottest and most evolved stars are consistent with these predictions. For HD 156623, we found that the acoustic-cutoff frequency is also the frequency of maximum power. This is similar to HD 34282, although $f_{\max }$ for HD 34282 is higher (79.423 day $^{-1}$; Casey et al. 2013). This implies-like HD 34282 - that HD 156623 should be consistent with the models and implications predicted by Casey (2011) and Aerts et al. (2010) and be consistent with the results of Zwintz et al. (2014b) if the age and evolutionary status estimations from Section 4.1 are correct.

To check this, we used the estimated $T_{\text {eff }}$ and $\log g$ from Table 3 and the acoustic-cutoff frequency $\left(71.143\right.$ day $^{-1}$ ) from Table 5 to plot HD 156623 (plotted as a black diamond) in a Kiel diagram alongside known pre-MS and early ZAMS $\delta$ Scutis from Zwintz et al. (2014b) in Figure 7. The five hottest pre-MS or early ZAMS $\delta$ Scutis from the sample by Zwintz et al. (2014b) - $\beta$ Pic (A6 V), HD 261711 (A2 V), HD 144277 (A1 V), HD 34282 (A0.5 V), and HD 37357 (A1 V) - have values of their highest excited frequency ranging between 70 and 90 day $^{-1}$. These are plotted in black instead of gray to highlight their similarity to HD 156623, which is the hottest star in the entire sample and its highest excited frequency is within this range. The theoretical blue edge (left dashed line) and the empirical red edge (right dashed line) of the classical $\delta$ Scuti instability strip as defined by Breger \& Pamyatnykh (1998) are also plotted. The highest excited frequency and HD 156623 's placement on the diagram were consistent with the other pre-MS or early ZAMS $\delta$ Scuti stars of the sample as predicted. This star also lies to the left of the theoretical blue edge of the classical instability strip (Breger \& Pamyatnykh 1998). It was noted that any of the higher candidate frequencies from Table 6, i.e., 75.211, 89.250, and 93.261 day ${ }^{-1}$, were also consistent with our interpretation.

\section{Conclusion}

The bRing observatories are the first to identify $\delta$ Scuti pulsations in the young A1 V star HD 156623, which hosts a gas-rich debris disk. We detected 16 frequencies in total, nine of which we confirmed to be real and the remaining seven we determined to be candidate frequencies. We found that the total amplitude from the nine confirmed frequencies was small and it is unsurprising that these pulsations were missed in previous studies of the star. We uncovered strong evidence of regularity between the frequencies. We found no evidence of frequency, amplitude, or phase modulation over the course of bRing's observations. The predicted $\log g$, along with our new estimated temperature and largest measured confirmed frequency, were used to compare HD 156623 to other pre-MS $\delta$ Scuti stars on a Kiel diagram with theoretical pre-MS and early ZAMS evolutionary tracks. HD 156623 is both consistent with similar stars and lies well within the predicted evolutionary tracks; it also lies just beyond the theoretical blue edge of the classical instability strip.

In addition to the frequency analysis, we performed a stellar characterization, spectral, and Sco-Cen membership analysis. We found HD 156623 to be a negligibly reddened $\sim 16 \pm 7 \mathrm{Myr}$ A1 V outlying member of the Sco-Cen subgroup Upper-Centaurus Lupus at $d \simeq 112 \mathrm{pc}$. The presence of the gas-rich debris disk supports a young age. 
Future work could include a full asteroseismic modeling similar to Zwintz et al. (2014a) and a more detailed abundance analysis of the star. Before such modeling is done, however, additional observations from bRing or other instruments should be made to verify the seven candidate frequencies reported in Table 6. These observations should also attempt to discover fainter frequencies. With this information and improved frequencies, this star will provide a better understanding of the young, hot $\delta$ Scutis.

S.N.M. is a U.S. Department of Defense SMART scholar sponsored by the U.S. Navy through SSC-LANT. The results reported herein benefited from collaborations and/or information exchange within NASA's Nexus for Exoplanet System Science (NExSS) research coordination network sponsored by NASA's Science Mission Directorate. Part of this research was carried out at the Jet Propulsion Laboratory, California Institute of Technology, under a contract with NASA. T.J.D. and E.E.M. acknowledge support from the Jet Propulsion Laboratory Exoplanetary Science Initiative. K.Z. acknowledges support by the Austrian Fonds zur Förderung der wissenschaftlichen Forschung (FWF, project V431-NBL) and the Austrian Space Application Programme (ASAP) of the Austrian Research Promotion Agency (FFG). The authors would like to acknowledge the support staff at both the South African Astronomical Observatory and Siding Spring Observatory for keeping both bRing stations maintained and running. Construction of the bRing observatory to be sited at Siding Springs, Australia would not be possible without a University of Rochester University Research Award, help from Mike Culver and Rich Sarkis (UR), and generous donations of time, services, and materials from Joe and Debbie Bonvissuto of Freight Expediters, Michael Akkaoui and his team at Tanury Industries, Robert Harris and Michael Fay at BCI, Koch Division, Mark Paup, Dave Mellon, and Ray Miller and the Zippo Tool Room. This work has made use of data from the European Space Agency (ESA) mission Gaia (https://www.cosmos.esa.int/gaia), processed by the Gaia Data Processing and Analysis Consortium (DPAC, https://www. cosmos.esa.int/web/gaia/dpac/consortium). Funding for the DPAC has been provided by national institutions, in particular the institutions participating in the Gaia Multilateral Agreement. This research was achieved using the POLLUX database (http://pollux.graal.univ-montp2.fr) operated at LUPM (Université Montpellier-CNRS, France with the support of the PNPS and INSU.

Facilities: bRing-SA, bRing-AU.

Software: Python 3.6.5 (Rossum 1995), scipy (Jones et al. 2001), matplotlib (Hunter 2007), numpy (van der Walt et al. 2011), astropy (The Astropy Collaboration et al. 2018), Period04 (Lenz \& Breger 2005), isoclassify (Huber et al. 2017), mwdust (Bovy et al. 2016).

\section{ORCID iDs}

Samuel N. Mellon (1) https://orcid.org/0000-0003-3405-2864 Eric E. Mamajek (1) https://orcid.org/0000-0003-2008-1488 Konstanze Zwintz (i) https://orcid.org/0000-0001-9229-8315 Trevor J. David (1) https://orcid.org/0000-0001-6534-6246 Remko Stuik (1) https://orcid.org/0000-0001-7797-3749 Geert Jan J. Talens (i) https://orcid.org/0000-0003-4787-2335 Patrick Dorval (i) https://orcid.org/0000-0003-3812-2436 Olivier Burggraaff (iD https://orcid.org/0000-0002-2487-4533
Matthew A. Kenworthy (iD https://orcid.org/0000-00027064-8270

John I. Bailey, III (D) https://orcid.org/0000-0002-4272-263X

Blaine B. D. Lomberg (iD https://orcid.org/0000-00021520-7851

Rudi B. Kuhn (ib https://orcid.org/0000-0002-4236-9020

Michael J. Ireland (1D https://orcid.org/0000-0002-6194-043X

Steven M. Crawford (iD https://orcid.org/0000-0002-

8969-5229

\section{References}

Aerts, C., Christensen-Dalsgaard, J., \& Kurtz, D. W. 2010, Asteroseismology (Cham: Springer)

Bailer-Jones, C. A. L., Rybizki, J., Fouesneau, M., Mantelet, G., \& Andrae, R. 2018, AJ, 156, 58

Bakos, G. Á., Lázár, J., Papp, I., Sári, P., \& Green, E. M. 2002, PASP, 114,974

Balona, L. A. 1994, MNRAS, 268, 119

Balona, L. A., \& Evers, E. A. 1999, MNRAS, 302, 349

Bertone, E., Buzzoni, A., Chávez, M., \& Rodríguez-Merino, L. H. 2004, AJ, 128,829

Bessell, M. S., Castelli, F., \& Plez, B. 1998, A\&A, 333, 231

Bovy, J., Rix, H.-W., Green, G. M., Schlafly, E. F., \& Finkbeiner, D. P. 2016, ApJ, 818, 130

Bowman, D. M., \& Kurtz, D. W. 2018, MNRAS, 476, 3169

Breger, M. 2000, BaltA, 9, 149

Breger, M., \& Pamyatnykh, A. A. 1998, A\&A, 332, 958

Brown, A. G. A., Vallenari, A., Prusti, T., et al. 2018, A\&A, 616, A1

Brown, A. G. A., \& Verschueren, W. 1997, A\&A, 319, 811

Burggraaff, O., Talens, G. J. J., Spronck, J., et al. 2018, A\&A, 617, A32

Capitanio, L., Lallement, R., Vergely, J. L., Elyajouri, M., \& Monreal-Ibero, A. 2017, A\&A, 606, A65

Cardelli, J. A., Clayton, G. C., \& Mathis, J. S. 1989, ApJ, 345, 245

Cargile, P. A., James, D. J., Pepper, J., et al. 2014, ApJ, 782, 29

Casey, M. P. 2011, PhD thesis, Saint Mary's Univ. Canada

Casey, M. P., Zwintz, K., Guenther, D. B., et al. 2013, MNRAS, 428, 2596

Castelli, F., \& Kurucz, R. L. 2004, arXiv:astro-ph/0405087

Castelli, F., \& Kurucz, R. L. 2006, A\&A, 454, 333

Choi, J., Dotter, A., Conroy, C., et al. 2016, ApJ, 823, 102

Cousins, A. W. J. 1976, MmRAS, 81, 25

Cousins, A. W. J. 1980, MNSSA, 39, 22

Cousins, A. W. J. 1983, SAAOC, 7, 36

Cousins, A. W. J. 1987, SAAOC, 11, 93

Cousins, A. W. J. 1990, SAAOC, 14, 55

Cousins, A. W. J., \& Stoy, R. H. 1962, RGOB, 49, 3

Crawford, D. L. 1958, ApJ, 128, 185

David, T. J., \& Hillenbrand, L. A. 2015, ApJ, 804, 146

de Zeeuw, P. T., Hoogerwerf, R., de Bruijne, J. H. J., Brown, A. G. A., \& Blaauw, A. 1999, AJ, 117, 354

Dotter, A. 2016, ApJS, 222, 8

ESA 1997, ESA SP-1200, The Hipparcos and Tycho Catalogues (Noordwijk: ESA) Fitch, W. S. 1981, ApJ, 249, 218

Fitzpatrick, E. L. 1999, PASP, 111, 63

Flower, P. J. 1996, ApJ, 469, 355

Gagné, J., Mamajek, E. E., Malo, L., et al. 2018, ApJ, 856, 23

Gaia Collaboration, Prusti, T., de Bruijne, J. H. J., et al. 2016, A\&A, 595, A1

Gray, D. F. 2005, The Observation and Analysis of Stellar Photospheres (Cambridge: Cambridge Univ. Press)

Green, G. M., Schlafly, E. F., Finkbeiner, D. P., et al. 2015, ApJ, 810, 25

Guenther, D. B., Kallinger, T., Zwintz, K., et al. 2009, ApJ, 704, 1710

Hartman, J. D., Bakos, G. Á., Kovács, G., \& Noyes, R. W. 2010, MNRAS, 408,475

Hartman, J. D., Gaudi, B. S., Holman, M. J., et al. 2008, ApJ, 675, 1254

Hauck, B., \& Mermilliod, M. 1997, yCat, 2215

Huber, D., Zinn, J., Bojsen-Hansen, M., et al. 2017, ApJ, 844, 102

Hunter, J. D. 2007, Matplotlib: A 2D Graphics Environment, http://www. scipy.org/

Jones, E., Oliphant, T., Peterson, P., et al. 2001, SciPy: Open source scientific tools for Python, http://www.scipy.org/

Kaufer, A., Stahl, O., Tubbesing, S., et al. 1999, Msngr, 95, 8

Kharchenko, N. V. 2001, KFNT, 17, 409

Kilkenny, D., \& Laing, J. D. 1992, MNRAS, 255, 308 
Kral, Q., Matrà, L., Wyatt, M. C., \& Kennedy, G. M. 2017, MNRAS, 469, 521 Kurtz, D. W., Saio, H., Takata, M., et al. 2014, MNRAS, 444, 102 Kurucz, R. L. 2005, MSAIS, 8, 14

Kuschnig, R., Weiss, W. W., Gruber, R., Bely, P. Y., \& Jenkner, H. 1997, A\&A, 328, 544

Lallement, R., Capitanio, L., Ruiz-Dern, L., et al. 2018, A\&A, 616, A132

Lenz, P., \& Breger, M. 2005, CoAst, 146, 53

Lieman-Sifry, J., Hughes, A. M., Carpenter, J. M., et al. 2016, ApJ, 828, 25

Mamajek, E. E., Meyer, M. R., \& Liebert, J. 2002, AJ, 124, 1670

Mamajek, E. E., Quillen, A. C., Pecaut, M. J., et al. 2012, AJ, 143, 72

Mamajek, E. E., Torres, G., Prsa, A., et al. 2015, arXiv:1510.06262

Marigo, P., Girardi, L., Bressan, A., et al. 2017, ApJ, 835, 77

McCall, M. L. 2004, AJ, 128, 2144

McDonald, I., Zijlstra, A. A., \& Boyer, M. L. 2012, MNRAS, 427, 343

Mellon, S., Stuik, R., Bailey, J., et al. 2018, AAS Meeting, 231, 152.22

Mellon, S. N., Mamajek, E. E., Oberst, T. E., \& Pecaut, M. J. 2017, ApJ, 844, 66

Menzies, J. W., Banfield, R. M., \& Laing, J. D. 1980, SAAOC, 1, 149

Menzies, J. W., Cousins, A. W. J., Banfield, R. M., \& Laing, J. D. 1989, SAAOC, 13, 1

Mermilliod, J.-C. 1997, yCat, 2168

Mermilliod, J.-C., Mermilliod, M., \& Hauck, B. 1997, A\&AS, 124, 349

Messina, S., Desidera, S., Turatto, M., Lanzafame, A. C., \& Guinan, E. F. 2010, A\&A, 520, A15

Montgomery, M. H., \& Odonoghue, D. 1999, DSSN, 13, 28

Moór, A., Curé, M., Kóspál, Á., et al. 2017, ApJ, 849, 123

Napiwotzki, R., Schoenberner, D., \& Wenske, V. 1993, A\&A, 268, 653

Oberst, T. E., Rodriguez, J. E., Colón, K. D., et al. 2017, AJ, 153, 97

Palacios, A., Gebran, M., Josselin, E., et al. 2010, A\&A, 516, A13

Paunzen, E. 2015, A\&A, 580, A23

Paunzen, E., Duffee, B., Heiter, U., Kuschnig, R., \& Weiss, W. W. 2001, A\&A, 373, 625

Pecaut, M. J., \& Mamajek, E. E. 2016, MNRAS, 461, 794

Pepper, J., Pogge, R. W., DePoy, D. L., et al. 2007, PASP, 119, 923

Pollacco, D. L., Skillen, I., Collier Cameron, A., et al. 2006, PASP, 118, 1407
Preibisch, T., \& Mamajek, E. 2008, in Handbook of Star Forming Regions, Volume II: The Southern Sky, Vol. 5, ed. B. Reipurth (San Francisco, CA: ASP), 235

Press, W. H., Teukolsky, S. A., Vetterling, W. T., \& Flannery, B. P. 1992, Numerical Recipes in FORTRAN. The Art of Scientific Computing (2nd ed.; Cambridge: Cambridge Univ. Press)

Ramella, M., Boehm, C., Gerbaldi, M., \& Faraggiana, R. 1989, A\&A, 209, 233

Rebollido, I., Eiroa, C., Montesinos, B., et al. 2018, A\&A, 614, A3

Rizzuto, A. C., Ireland, M. J., \& Zucker, D. B. 2012, MNRAS, 421, L97

Rossum, G. 1995, Python Reference Manual (Amsterdam: CWI), https://docs. python.org $/ 2.0 /$ ref/ref.html

Royer, F., Gebran, M., Monier, R., et al. 2014, A\&A, 562, A84

Royer, F., Grenier, S., Baylac, M.-O., Gómez, A. E., \& Zorec, J. 2002, A\&A, 393,897

Scargle, J. D. 1982, ApJ, 263, 835

Slawson, R. W., Hill, R. J., \& Landstreet, J. D. 1992, ApJS, 82, 117

Snellen, I., Stuik, R., Otten, G., et al. 2013, Eur. Phys. J. Web Conf., 47, 03008

Snellen, I. A. G., Stuik, R., Navarro, R., et al. 2012, Proc. SPIE, 8444, 84440I

Stuik, R., Bailey, J. I., Dorval, P., et al. 2017, A\&A, 607, A45

Stuik, R., Lesage, A.-L., Jakobs, A., Spronck, J. F. P., \& Snellen, I. A. G. 2014, Proc. SPIE, 9152, 91520N

Talens, G. J. J., Deul, E. R., Stuik, R., et al. 2018, A\&A, 619, A154

Talens, G. J. J., Spronck, J. F. P., Lesage, A.-L., et al. 2017, A\&A, 601, A11

The Astropy Collaboration, Price-Whelan, A. M., Sipocz, B. M., et al. 2018, AJ, 156, 123

Torres, G. 2010, AJ, 140, 1158

van der Walt, S., Colbert, S. C., \& Varoquaux, G. 2011, The NumPy Array: A Structure for Efficient Numerical Computation, http://www.scipy.org/ van Leeuwen, F. 2007, A\&A, 474, 653

Wang, J. J., Graham, J. R., Pueyo, L., et al. 2016, AJ, 152, 97

Watson, C. L., Henden, A. A., \& Price, A. 2006, SASS, 25, 47

Zwintz, K., Fossati, L., Ryabchikova, T., et al. 2014b, Sci, 345, 550

Zwintz, K., Lenz, P., Breger, M., et al. 2011, A\&A, 533, A133

Zwintz, K., Ryabchikova, T., Lenz, P., et al. 2014a, A\&A, 567, A4 